\title{
Evaluation of Psychometric Properties of the Persian Version of the Predicaments Questionnaire, Exploring Social Attitudes to Suicide
}

\section{Ali Rafati}

Iran University of Medical Sciences

Leila Janani

Iran University of Medical Sciences

Seyed Kazem Malakouti

Iran University of Medical Sciences

Seyed Abbas Motevalian

Iran University of Medical Sciences

\section{Ali Kabiri}

Iran University of Medical Sciences

\section{Yeganeh Pasebani}

Iran University of Medical Sciences

Mohammadreza Shalbafan ( $\square$ Shalbafan.mr@iums.ac.ir)

Iran University of Medical Sciences https://orcid.org/0000-0002-4536-3441

\section{Research Article}

Keywords: psychometrics, reproducibility of results, surveys and questionnaires, suicide

Posted Date: January 28th, 2022

DOI: https://doi.org/10.21203/rs.3.rs-1226165/v1

License: (c) (i) This work is licensed under a Creative Commons Attribution 4.0 International License. Read Full License 


\section{Abstract}

Suicide is the second leading cause of death between the ages of 15 and 29. Most suicides are related to mental illnesses. Suicide is considered a response to predicaments. Due to the growing prevalence of suicide, assessing people's attitudes toward suicide is necessary. Therefore, this study aimed to examine the psychometric properties of the Persian version of the Predicaments Questionnaire (PQ), measuring social attitudes toward suicide.

\section{Methods:}

This psychometrics study evaluated face validity, content validity, temporal stability, internal consistency, and exploratory factor analysis. First, the questionnaire was translated into Persian by the translate-backtranslate method. The Persian version was provided to 10 experts in psychiatry for further revision. Two indicators, CVR and CVI, were calculated to evaluate the content validity. To check the face validity, we prepared a form and gave it to 10 people outside the campus to submit their opinions. The results were then given to 10 experts to comment on.

Temporal stability was investigated by the test-retest method, reporting Intraclass correlation (ICC). Internal consistency was assessed by reporting Cronbach's alpha and McDonald's Omega coefficients. Exploratory factor analysis was used to determine the number of dimensions of the questionnaire. In this analysis, $\chi 2, T L I, C F I, R M S E A$, and SRMR indices were calculated. All analyses were performed using SPSS software version 22 and $R$ software version 4.

\section{Results:}

A total of 151 students were enrolled with a mean age of $25(S D=0.32)$. The Persian $P Q$ was valid in terms of content validity and face validity. Furthermore, it was reliable as Cronbach's alpha, McDonald's Omega, and the ICC were $0.94,0.943$, and 0.998 , respectively. In addition, the exploratory factor analysis yielded one dimension. Finally, after reviewing the experts' comments, the final amendments were made, and only question 29 was removed from the final version.

\section{Conclusion:}

Consequently, the Persian version of the $\mathrm{PQ}$ is acceptable in terms of content validity, face validity, temporal stability, and internal consistency. This questionnaire can be used in future research on Persianspeaking society.

\section{Background:}

Suicide means deliberately taking one's own life, and if it is complete, it means a deadly act to achieve one's desire for death [1]. Every 40 seconds, one person in the world dies due to suicide, which numbers 800,000 a year. Suicide is known as the second leading cause of death between the ages of 15 and 29. For every suicide resulting in death, more than 20 others attempt suicide [2]. The suicide rate in 2012 in 
the world was estimated to be 12 out of every 100,000, accounting for 793,000 deaths in 2016 [3]. This amount was 5.3 per 100,000 for Iran $[4,5]$. Approximately $1.4 \%$ of all deaths result from suicide worldwide $[3,6]$. The rate of complete suicide is higher in men than women, ranging from 1.5 times higher in developing countries to 3.5 times higher in developed countries. Suicide is generally prevalent among people over 70 years of age. However, in some countries, people between 15 and 30 are at higher risk [2]. Ten to twenty million non-lethal suicides occur each year [7]. Non-lethal suicide attempts can lead to longterm injuries and disabilities. In the Western world, there are more suicide attempts among young people and women [8]. Common methods of suicide include hanging, pesticide poisoning, and weapons [9].

The suicide rate in Iran is lower than the world average, still higher than the Middle East average. The most prevalent method of suicide in Iran is hanging in men and burning in women. [4, 10]. Although women attempt more suicides than men, they attempt fewer completed suicides than men, reflecting the difference in the method of suicide between them [11].

In Iran, the highest suicide rate for men is observed in Lorestan, Hamedan, and Ilam provinces and for women in Lorestan and Kermanshah provinces [10]. As a result, llam, Kermanshah, Lorestan, and Hamedan provinces have the highest suicide mortality rates in Iran. One reason for such a high suicide rate might be due to the low socioeconomic status. On the other hand, these provinces have the country's highest unemployment and divorce rates. Finally, the tribal structure and extreme fanaticism could be other potential reasons [12].

The most common risk factors for suicide are mental illnesses (including depression, bipolar disorder, autism, schizophrenia, personality disorders, anxiety disorders, and substance abuse), relationship problems, employment and financial hardships, and history of suicide attempts [13-15]. However, certain suicides are impulsive and abrupt reactions to stress, marital problems, or rape. People with a history of suicide are at higher risk of attempting suicide. Practical suicide prevention efforts include accurate media coverage of suicide, economic improvement, and restricting access to weapons, poisons, and drugs. Common methods of suicide vary in different regions and countries and depend on the availability of these methods in these regions [16].

It has been shown, broad existential themes, such as religion, honour, and life's meaning, have influenced perspectives on suicide [17]. The Abrahamic religions, for instance, traditionally consider suicide a crime against God, believing in life's sanctity. In this regard, in some countries, suicide is widely regarded as a criminal offence [18]. In the 20th and 21st centuries, suicide has rarely been utilised as a means of protest. Moreover, suicide bombings as a terrorist tactic have been observed [19]. Suicide is often considered a major disaster and is regarded as an adverse action almost globally.

Numerous studies have examined communities' attitudes toward suicide [20]. A major challenge in measuring people's attitudes toward suicide is their subjectivity and variability over time. A significant number of these measuring instruments contain suicide myths, i.e., society's misconceptions about suicide. According to the World Health Organization, one of these myths and misconceptions is the belief that "only people with mental disorders attempt suicide." Other myths include: Talking about suicide 
encourages it; most suicides occur without warning; people who attempt suicide do not talk about it; people who attempt suicide are determined to die [21].

The Predicaments Questionnaire designed by Shahtahmasebi et al. in December 2016 measures the social attitude toward suicide in all individuals, regardless of any social or socioeconomic classes. It also removes the questionnaire from suicide myths. This study considers suicide as a response to predicaments, and it demonstrates that the questionnaire's scores have a direct relationship with the suicide rate in a group or population [22]. Multi-centre and international studies in this field are highly required. Therefore, this study aimed to examine the psychometric properties of the Persian translation of the "Social Attitudes to Suicide dealing with Predicaments" Questionnaire.

\section{Methods:}

This is a psychometric research performed at the Iran University of Medical Sciences in 2020. The analysis included face validity, content validity, temporal stability reliability, internal consistency reliability, and construct validity. First, with the questionnaire designer's permission, the questionnaire was received via email, and then it was translated into Persian by the translate-back-translate method [23]. For this purpose, we recruited two independent translators to translate it into Persian and two other independent translators to translate it again into English. Afterwards, we prepared the questionnaire's initial Persian version by examining the Persian and English translations' semantic compatibility and the original English version. We provided ten experts in psychiatry with the Persian version to complete and modify the questionnaire by reviewing, discussing, exchanging opinions, and checking cultural adaptation.

We calculated two indicators, the Content Validity Ratio (CVR) [24] and the Content Validity Index (CVI) [25], to evaluate the content validity. For obtaining CVR, we asked experts to categorise each question based on the essentialness. For calculating $\mathrm{CVI}$, they specified relevance, clarity, and simplicity for each question. Based on the number of specialists (10 in this study), the minimum accepted CVR score is 0.62. The minimum acceptable score for a CVI is 0.79 [25].

To check the face validity, we prepared a form and gave it to 10 people outside the campus to submit their opinions. The results were then given to 10 experts to comment on. These ten included psychiatrists, psychologists, and epidemiologists from the Iran University of Medical Sciences. We subsequently asked them to score (using the 5-state Likert-type scale of 1 to 5 ) the importance of each question. Then we calculated the impact score of each question using the following formula: Impact score= Frequency (\%) of the experts scoring 4 or $5 \times$ mean score of the question. The minimum acceptable impact score is more than 1.5 [26].

In the reliability analysis, we examined two areas: temporal stability and internal consistency. First, we evaluated the temporal stability by the test-retest method. For this purpose, 30 people completed the questionnaire in a pilot study. Then two weeks later, they completed it again. We reported the correlation between the two tests' scores using the Intraclass Correlation Coefficient (ICC). The internal consistency is checked by reporting Cronbach's alpha coefficient and McDonald's Omega coefficient. One hundred 
fifty-one students of the Iran University of Medical Sciences completed the questionnaire. We calculated Cronbach's alpha coefficient and McDonald Omega coefficient by analysing the collected data.

We evaluated construct validity by confirmatory factor analysis to confirm the one-dimensionality of the questionnaire. In this analysis, we measured $\chi 2$, the Root Mean Square Error of Approximation (RMSR), Comparative Fit Index (CFI), Tucker-Lewis index (TLI), and Standardised Root Mean Square Residual (SRMR).

\section{Research population and Sampling method:}

We performed this study on the Iran University of Medical Sciences students with ten faculty members in psychiatry, psychology, and epidemiology as experts. The experts evaluated the face and the content validities. To check the internal consistency, we selected a random sample of 200 people from the Iran University of Medical Sciences school of medicine. The questionnaire was emailed to or sent to them as a text message, and subsequently, 151 of them responded and filled out the questionnaire. 30 of the 151 students refilled the forms two weeks later to test-retest.

\section{Methods and tools of data collection:}

The study questionnaire, called the Predicament Questionnaire, was prepared by Shahtahmasebi et al. in 2016. The questionnaire consists of 41 items. The first nine items record demographic information and personal information, including religion, history of suicide, attitudes toward suicide, depression, hopelessness, and lack of interest. The following 32 items describe scenarios and ask whether the person has the right to attempt suicide in those circumstances. The most common predicaments discussed in this study include the onset of a terminal illness, becoming wheelchair-dependent, severe mental disorders, and car crashes while drunk. For each question, the respondent chooses from a Likert-type scale of 1 to 4 , of which 1 is the lowest value and 4 is the highest.

Here is a sample of the questions of the studied instrument:

"Person $\mathrm{C}$ was driving below the speed limit on a suburban street. A child ran onto the road. To avoid the child, person $\mathrm{C}$ swerved and killed an adult on the other footpath.

Would person $\mathrm{C}$ have suicidal thoughts?

1. No 2.Slight 3. Moderate 4.Strong."

This questionnaire was shown to measure only one factor. Moreover, it was indicated to have a high Cronbach's Alpha coefficient showing a high internal consistency [22].

\section{Method of analysis:}

We Calculated CVR, CVI, impact score, Cronbach's alpha, and ICC in SPSS software version 22 and performed confirmatory factor analysis and McDonald's Omega in R software version 4. 


\section{Results:}

Of the 151 participants, 42 (27.8\%) were male, and 109 (72.2\%) were female. In terms of age, all participants were in the range of 20 to 30 years old. In terms of marital status, 81 were single (53.6\%), 56 were married (37.1\%), five were divorced (3.3\%), and nine were uncategorised (6\%) (Table 1). The frequency of the responses is shown in Table 2. The respondents' mean questionnaire score was 61.98 $(S D=1.45)$ out of a maximum score of 128 . Furthermore, we calculated the mean scores by sex and marital status as follows: $60.78(S D=18.02)$ and $62.45(S D=17.89)$ for men and women, respectively, and 65.53 (SD=16.12), 58.15 (SD=19.38), and $54.40(S D=14.63)$ for the single, the married, and the divorced, respectively.

Table 1

Demographic data

\begin{tabular}{|ll|}
\hline Age, $\mathbf{M}(\mathrm{SD})$ & $\mathbf{2 5}(\mathbf{S D}=\mathbf{0 . 3 2})$ \\
\hline Sex, $\mathrm{n}(\%)$ & Female: $109(72.2 \%)$ \\
\cline { 2 - 2 } & Male: $42(27.8 \%)$ \\
\hline Marital status, $\mathrm{n}(\%)$ & Single: $81(53.6 \%)$ \\
& $\begin{array}{l}\text { Married: } 56(37.1 \%) \\
\text { Divorced: } 5(3.3 \%)\end{array}$ \\
& Others: $9(6 \%)$ \\
\hline
\end{tabular}


Table 2

Frequency of the responses

No. Frequency

Median $\quad M(S D)$

No (1) Slight (2) Moderate (3) Strong (4)

\begin{tabular}{|c|c|c|c|c|c|c|}
\hline 1 & $82(54.3 \%)$ & 40 (26.5\%) & $26(17.2 \%)$ & $3(2 \%)$ & 1.00 & $1.67(0.83)$ \\
\hline 2 & $70(46.4 \%)$ & $54(35.8 \%)$ & 19 (12.6\%) & $8(5.3 \%)$ & 2.00 & $1.77(0.87)$ \\
\hline 3 & 77 (51\%) & 37 (24.5\%) & 29 (19.2\%) & $8(5.3 \%)$ & 1.00 & $1.79(0.93)$ \\
\hline 4 & 78 (51.7\%) & 39 (25.8\%) & $28(18.5 \%)$ & $6(4 \%)$ & 1.00 & $1.75(0.90)$ \\
\hline 5 & $48(31.8 \%)$ & $36(23.8 \%)$ & 44 (29.1\%) & $23(15.2 \%)$ & 2.00 & $2.28(1.10)$ \\
\hline 6 & $25(16.6 \%)$ & 31 (20.5\%) & 39 (25.8\%) & $56(37.1 \%)$ & 3.00 & $2.83(1.14)$ \\
\hline 7 & 27 (17.9\%) & 25 (16.6\%) & 35 (23.2\%) & 64 (42.4\%) & 3.00 & $2.90(1.21)$ \\
\hline 8 & 28 (18.5\%) & 21 (13.9\%) & 44 (29.1\%) & 58 (38.4\%) & 3.00 & $2.87(1.21)$ \\
\hline 9 & 49 (32.5\%) & 25 (16.6\%) & $34(22.5 \%)$ & 43 (28.5\%) & 3.00 & $2.50(1.21)$ \\
\hline 10 & 73 (48.3\%) & $43(28.5 \%)$ & 30 (19.9\%) & 5 (3.3\%) & 2.00 & $1.78(0.88)$ \\
\hline 11 & $74(49.0 \%)$ & 44 (29.1\%) & 25 (16.6\%) & $8(5.3 \%)$ & 2.00 & $1.78(0.91)$ \\
\hline 12 & $86(57.0 \%)$ & $44(29.1 \%)$ & $21(13.9 \%)$ & $0(0 \%)$ & 1.00 & $1.57(0.73)$ \\
\hline 13 & 88 (58.3\%) & $36(23.8 \%)$ & $23(15.2 \%)$ & 4 (2.6\%) & 1.00 & $1.62(0.84)$ \\
\hline 14 & 105 (69.5\%) & 29 (19.2\%) & 15 (9.9\%) & $2(1.3 \%)$ & 1.00 & $1.43(0.73)$ \\
\hline 15 & 98 (64.9\%) & $26(17.2 \%)$ & $20(13.2 \%)$ & 7 (4.6\%) & 1.00 & $1.58(0.89)$ \\
\hline 16 & 25 (16.6\%) & 38 (25.2\%) & 51 (33.8\%) & 37 (24.5\%) & 3.00 & $2.66(1.03)$ \\
\hline 17 & 93 (61.6\%) & 36 (23.8\%) & 15 (9.9\%) & $7(4.6 \%)$ & 1.00 & $1.58(0.85)$ \\
\hline 18 & $28(18.5 \%)$ & 30 (19.9\%) & 51 (33.8\%) & 42 (27.8\%) & 3.00 & $2.71(1.07)$ \\
\hline 19 & $126(83.4 \%)$ & 16 (10.6\%) & $8(5.3 \%)$ & $1(0.7 \%)$ & 1.00 & $1.23(0.57)$ \\
\hline 20 & $67(44.4 \%)$ & 53 (35.1\%) & 21 (13.9\%) & 10 (6.6\%) & 2.00 & $1.83(0.91)$ \\
\hline 21 & 129 (85.4\%) & 16 (10.6\%) & $6(4.0 \%)$ & $0(0 \%)$ & 1.00 & $1.19(0.48)$ \\
\hline 22 & 100 (66.2\%) & 33 (21.9\%) & $11(7.3 \%)$ & $7(4.6 \%)$ & 1.00 & $1.50(0.82)$ \\
\hline 23 & 60 (39.7\%) & 43 (28.5\%) & 34 (22.5\%) & 14 (9.3\%) & 2.00 & $2.01(1.00)$ \\
\hline 24 & 61 (40.4\%) & 53 (35.1\%) & $26(17.2 \%)$ & 11 (7.3\%) & 2.00 & $1.91(0.93)$ \\
\hline 25 & 44 (29.1\%) & 39 (25.8\%) & 40 (26.5\%) & 28 (18.5\%) & 2.00 & 2.34 (1.09) \\
\hline 26 & 37 (24.5\%) & $32(21.2 \%)$ & 36 (23.8\%) & 46 (30.5\%) & 3.00 & $2.60(1.16)$ \\
\hline
\end{tabular}




\begin{tabular}{|c|c|c|c|c|c|c|}
\hline \multirow[t]{2}{*}{ No. } & \multicolumn{4}{|l|}{ Frequency } & \multirow[t]{2}{*}{ Median } & \multirow[t]{2}{*}{$M(S D)$} \\
\hline & No (1) & Slight (2) & Moderate (3) & Strong (4) & & \\
\hline 27 & 64 (42.4\%) & 38 (25.2\%) & 30 (19.9\%) & 19 (12.6\%) & 2.00 & $2.03(1.06)$ \\
\hline 28 & $58(38.4 \%)$ & 29 (19.2\%) & $39(25.8 \%)$ & $25(16.6 \%)$ & 2.00 & $2.21(1.13)$ \\
\hline 29 & 116 (76.7\%) & $14(9.3 \%)$ & $12(7.9 \%)$ & $9(6 \%)$ & 1.00 & $1.43(0.876)$ \\
\hline 30 & $45(29.8 \%)$ & 25 (16.6\%) & $33(21.9 \%)$ & $48(31.8)$ & 3.00 & $2.56(1.22)$ \\
\hline 31 & $72(47.7 \%)$ & 34 (22.5\%) & $29(19.2 \%)$ & $16(10.6 \%)$ & 2.00 & $1.93(1.04)$ \\
\hline 32 & $90(59.6 \%)$ & $34(22.5 \%)$ & $23(15.2 \%)$ & $4(2.6 \%)$ & 1.00 & $1.61(0.84)$ \\
\hline
\end{tabular}

\section{Validity Results:}

According to the content validity results, questions 17, 19, 21, 23, 29, 31, and 32 lacked the required CVR score. Furthermore, questions 19, 29, and 32 did not have the required CVI score. Also, all questions were accepted in terms of simplicity and clarity. The average CVI of the questionnaire is 0.95 . We assessed face validity using quantitative and qualitative methods. In a quantitative study, we measured each question's importance using a 5-state Likert-type scale, and the impact score of each question was determined. According to the analysis results, questions 4, 19, 21, and 29 lacked the required impact score.

In the qualitative method, we asked experts' opinions about each question. They mentioned the problems, ambiguities, inadequacies in the meaning, and the shortcomings of questions 19 and 20, which had writing problems, and were subsequently modified. Finally, after reviewing the results and discussing the experts' opinions, only question 29 was invalid and unreliable and removed from the questionnaire. The results of this study can be seen in Table 3 . 
Table 3

Validity results in the Persian version of the Predicaments Questionnaire

\begin{tabular}{|c|c|c|c|c|c|c|}
\hline No. & & CVR & $\begin{array}{l}\text { CVI } \\
\text { (Relevance) }\end{array}$ & $\begin{array}{l}\text { CVI } \\
\text { (Simplicity) }\end{array}$ & $\begin{array}{l}\text { CVI } \\
\text { (Clarity) }\end{array}$ & $\begin{array}{l}\text { Impact } \\
\text { score }\end{array}$ \\
\hline \multirow[t]{3}{*}{1} & $\begin{array}{l}\text { Person A had a romantic } \\
\text { relationship of about } 1 \text { year, with } \\
\text { person B, but they had not been } \\
\text { living together. Person B ended the } \\
\text { relationship and commenced a } \\
\text { new relationship with a third } \\
\text { person. }\end{array}$ & 1 & 0.9 & 1 & 1 & 2.73 \\
\hline & $\begin{array}{l}\text { Would person A have suicidal } \\
\text { thoughts? }\end{array}$ & & & & & \\
\hline & 1. No 2.Slight 3.Moderate 4.Strong & & & & & \\
\hline \multirow[t]{3}{*}{2} & $\begin{array}{l}\text { Person A had a romantic } \\
\text { relationship with person B of about } \\
1 \text { year, and they had been living } \\
\text { together. Person B ended the } \\
\text { relationship and commenced a } \\
\text { new relationship with a third } \\
\text { person. }\end{array}$ & 1 & 0.9 & 1 & 0.9 & 3.51 \\
\hline & $\begin{array}{l}\text { Would person A have suicidal } \\
\text { thoughts? }\end{array}$ & & & & & \\
\hline & 1. No 2.Slight 3.Moderate 4.Strong & & & & & \\
\hline \multirow[t]{3}{*}{3} & $\begin{array}{l}\text { Person } A \text { and Person } B \text { had been } \\
\text { married for about } 1 \text { year. Person } B \\
\text { ended the marriage and } \\
\text { commenced a new relationship } \\
\text { with a third person. }\end{array}$ & 0.8 & 0.8 & 1 & 1 & 1.85 \\
\hline & $\begin{array}{l}\text { Would person A have suicidal } \\
\text { thoughts? }\end{array}$ & & & & & \\
\hline & 1. No 2.Slight 3.Moderate 4.Strong & & & & & \\
\hline \multirow[t]{3}{*}{4} & $\begin{array}{l}\text { Person C was driving below the } \\
\text { speed limit on a suburban street. A } \\
\text { child ran onto the road. To avoid } \\
\text { the child, person C swerved and } \\
\text { killed an adult on the other } \\
\text { footpath. }\end{array}$ & 0.7 & 0.7 & 0.9 & 1 & 1.28 \\
\hline & $\begin{array}{l}\text { Would person } \mathrm{C} \text { have suicidal } \\
\text { thoughts? }\end{array}$ & & & & & \\
\hline & 1. No 2.Slight 3.Moderate 4.Strong & & & & & \\
\hline
\end{tabular}




\begin{tabular}{|c|c|c|c|c|c|c|}
\hline No. & & CVR & $\begin{array}{l}\text { CVI } \\
\text { (Relevance) }\end{array}$ & $\begin{array}{l}\text { CVI } \\
\text { (Simplicity) }\end{array}$ & $\begin{array}{l}\text { CVI } \\
\text { (Clarity) }\end{array}$ & $\begin{array}{l}\text { Impact } \\
\text { score }\end{array}$ \\
\hline 5 & $\begin{array}{l}\text { Person C attended a party and got } \\
\text { drunk. In spite of advice not to } \\
\text { drive, and the offer of being driven } \\
\text { home by a friend, person C insisted } \\
\text { on driving. Person C drove above } \\
\text { the speed limit and hit and killed a } \\
\text { pedestrian on a pedestrian } \\
\text { crossing. } \\
\text { Would person C have suicidal } \\
\text { thoughts? } \\
\text { 1. No 2.Slight 3.Moderate 4.Strong }\end{array}$ & 1 & 0.9 & 1 & 1 & 3.3 \\
\hline 6 & $\begin{array}{l}\text { Person } D \text { has heterosexual } \\
\text { intercourse with Person } Z \text {. Without } \\
\text { Person D's permission, this event is } \\
\text { secretly filmed and placed on the } \\
\text { web by a third person. } \\
\text { Would person D have suicidal } \\
\text { thoughts? } \\
\text { 1. No 2.Slight 3.Moderate 4.Strong }\end{array}$ & 1 & 1 & 1 & 1 & 3.87 \\
\hline 7 & $\begin{array}{l}\text { Person D has homosexual } \\
\text { intercourse with Person Y. Without } \\
\text { Person D's permission, this event is } \\
\text { secretly filmed and placed on the } \\
\text { web by a third person. } \\
\text { Would person D have suicidal } \\
\text { thoughts? } \\
\text { 1. No 2.Slight 3.Moderate 4.Strong }\end{array}$ & 1 & 1 & 1 & 1 & 3.96 \\
\hline 8 & $\begin{array}{l}\text { Person E suffers spinal injuries and } \\
\text { will be confined to a wheelchair for } \\
\text { life. } \\
\text { Would person E have suicidal } \\
\text { thoughts? } \\
\text { 1. No 2.Slight 3.Moderate 4.Strong }\end{array}$ & 1 & 1 & 1 & 1 & 4.6 \\
\hline 9 & $\begin{array}{l}\text { Person E develops a painful, } \\
\text { terminal (will be fatal) disorder. } \\
\text { Would person E have suicidal } \\
\text { thoughts? } \\
\text { 1. No 2.Slight 3.Moderate 4.Strong }\end{array}$ & 1 & 0.9 & 1 & 1 & 4.6 \\
\hline
\end{tabular}




\begin{tabular}{|c|c|c|c|c|c|c|}
\hline No. & & CVR & $\begin{array}{l}\text { CVI } \\
\text { (Relevance) }\end{array}$ & $\begin{array}{l}\text { CVI } \\
\text { (Simplicity) }\end{array}$ & $\begin{array}{l}\text { CVI } \\
\text { (Clarity) }\end{array}$ & $\begin{array}{l}\text { Impact } \\
\text { score }\end{array}$ \\
\hline \multirow[t]{3}{*}{10} & $\begin{array}{l}\text { Person F comes from a very high } \\
\text { status and well-educated family. } \\
\text { Person F is convicted of stealing } \\
\text { and jailed. }\end{array}$ & 0.8 & 0.8 & 0.9 & 0.9 & 2.22 \\
\hline & $\begin{array}{l}\text { Would person } \mathrm{F} \text { have suicidal } \\
\text { thoughts? }\end{array}$ & & & & & \\
\hline & 1. No 2.Slight 3.Moderate 4.Strong & & & & & \\
\hline \multirow[t]{3}{*}{11} & $\begin{array}{l}\text { Person F comes from a very high } \\
\text { status and well-educated family. } \\
\text { Person F studies very hard, but } \\
\text { lacks academic skills and at the } \\
\text { end of a year at university, fails } \\
\text { every subject. }\end{array}$ & 0.8 & 1 & 1 & 1 & 3.36 \\
\hline & $\begin{array}{l}\text { Would person } \mathrm{F} \text { have suicidal } \\
\text { thoughts? }\end{array}$ & & & & & \\
\hline & 1. No 2.Slight 3.Moderate 4.Strong & & & & & \\
\hline \multirow[t]{3}{*}{12} & $\begin{array}{l}\text { Person } G \text { comes from an average } \\
\text { family. Person } G \text { is convicted of } \\
\text { stealing and jailed. }\end{array}$ & 1 & 0.9 & 0.7 & 0.9 & 2.66 \\
\hline & $\begin{array}{l}\text { Would person } \mathrm{G} \text { have suicidal } \\
\text { thoughts? }\end{array}$ & & & & & \\
\hline & 1. No 2.Slight 3.Moderate 4.Strong & & & & & \\
\hline \multirow[t]{3}{*}{13} & $\begin{array}{l}\text { Person G comes from an average } \\
\text { family. Person G studies very hard, } \\
\text { but lacks academic skills and at } \\
\text { the end of a year at university, fails } \\
\text { every subject. }\end{array}$ & 0.8 & 1 & 1 & 0.9 & 3.2 \\
\hline & $\begin{array}{l}\text { Would person G have suicidal } \\
\text { thoughts? }\end{array}$ & & & & & \\
\hline & 1. No 2.Slight 3.Moderate 4.Strong & & & & & \\
\hline \multirow[t]{3}{*}{14} & $\begin{array}{l}\text { Person } \mathrm{H} \text { and Person } \mathrm{X} \text { lived in the } \\
\text { same street as children and have } \\
\text { been life-long, close friends. Person } \\
\mathrm{H} \text { is killed in a train crash. }\end{array}$ & 0.8 & 0.7 & 1 & 1 & 2.04 \\
\hline & $\begin{array}{l}\text { Would person } \mathrm{X} \text { have suicidal } \\
\text { thoughts? }\end{array}$ & & & & & \\
\hline & 1. No 2.Slight 3.Moderate 4.Strong & & & & & \\
\hline
\end{tabular}




\begin{tabular}{|c|c|c|c|c|c|c|}
\hline No. & & CVR & $\begin{array}{l}\text { CVI } \\
\text { (Relevance) }\end{array}$ & $\begin{array}{l}\text { CVI } \\
\text { (Simplicity) }\end{array}$ & $\begin{array}{l}\text { CVI } \\
\text { (Clarity) }\end{array}$ & $\begin{array}{l}\text { Impact } \\
\text { score }\end{array}$ \\
\hline \multirow[t]{3}{*}{15} & $\begin{array}{l}\text { Person } \mathrm{H} \text { and Person } \mathrm{X} \text { lived in the } \\
\text { same street as children and have } \\
\text { been life-long, close friends. Person } \\
\mathrm{H} \text { kills him/herself by standing in } \\
\text { the path of a train. }\end{array}$ & 0.8 & 1 & 1 & 1 & 3.28 \\
\hline & $\begin{array}{l}\text { Would person } \mathrm{X} \text { have suicidal } \\
\text { thoughts? }\end{array}$ & & & & & \\
\hline & 1. No 2.Slight 3.Moderate 4.Strong & & & & & \\
\hline \multirow[t]{3}{*}{16} & $\begin{array}{l}\text { Person } J \text { dropped a gas bottle } \\
\text { which exploded. Person J } \\
\text { sustained severe burns to the face } \\
\text { and hands, which left disfiguring } \\
\text { scars. }\end{array}$ & 1 & 1 & 1 & 1 & 4.5 \\
\hline & $\begin{array}{l}\text { Would person J have suicidal } \\
\text { thoughts? }\end{array}$ & & & & & \\
\hline & 1. No 2.Slight 3.Moderate 4.Strong & & & & & \\
\hline \multirow[t]{3}{*}{17} & $\begin{array}{l}\text { Person } \mathrm{K} \text { developed a mental } \\
\text { disorder which responds well to } \\
\text { treatment, and does not cause } \\
\text { Person } \mathrm{K} \text { to lose more than } 5 \\
\text { working days per year. }\end{array}$ & 0.6 & 0.9 & 0.9 & 0.9 & 2.1 \\
\hline & $\begin{array}{l}\text { Would person } \mathrm{K} \text { have suicidal } \\
\text { thoughts? }\end{array}$ & & & & & \\
\hline & 1. No 2.Slight 3.Moderate 4.Strong & & & & & \\
\hline \multirow[t]{3}{*}{18} & $\begin{array}{l}\text { Person } \mathrm{K} \text { develops a mental } \\
\text { disorder, which does not respond } \\
\text { well to treatment, and Person } \mathrm{K} \text { is } \\
\text { no longer able to work. }\end{array}$ & 1 & 1 & 1 & 0.9 & 3.87 \\
\hline & $\begin{array}{l}\text { Would person } \mathrm{K} \text { have suicidal } \\
\text { thoughts? }\end{array}$ & & & & & \\
\hline & 1. No 2.Slight 3.Moderate 4.Strong & & & & & \\
\hline \multirow[t]{3}{*}{19} & $\begin{array}{l}\text { Person } \mathrm{K} \text { develops arthritis, which } \\
\text { responds well to treatment, and } \\
\text { does not cause Person } \mathrm{K} \text { to lose } \\
\text { more than } 5 \text { working days per year. }\end{array}$ & 0.4 & 0.5 & 0.8 & 1 & 1.12 \\
\hline & $\begin{array}{l}\text { Would person } \mathrm{K} \text { have suicidal } \\
\text { thoughts? }\end{array}$ & & & & & \\
\hline & 1. No 2.Slight 3.Moderate 4.Strong & & & & & \\
\hline
\end{tabular}




\begin{tabular}{|c|c|c|c|c|c|c|}
\hline No. & & CVR & $\begin{array}{l}\text { CVI } \\
\text { (Relevance) }\end{array}$ & $\begin{array}{l}\text { CVI } \\
\text { (Simplicity) }\end{array}$ & $\begin{array}{l}\text { CVI } \\
\text { (Clarity) }\end{array}$ & $\begin{array}{l}\text { Impact } \\
\text { score }\end{array}$ \\
\hline \multirow[t]{3}{*}{20} & $\begin{array}{l}\text { Person } \mathrm{K} \text { develops arthritis, which } \\
\text { does not respond well to treatment, } \\
\text { and Person } \mathrm{K} \text { is no longer able to } \\
\text { work. }\end{array}$ & 0.8 & 0.8 & 0.9 & 0.9 & 1.75 \\
\hline & $\begin{array}{l}\text { Would person } \mathrm{K} \text { have suicidal } \\
\text { thoughts? }\end{array}$ & & & & & \\
\hline & 1. No 2.Slight 3.Moderate 4.Strong & & & & & \\
\hline \multirow[t]{3}{*}{21} & $\begin{array}{l}\text { Person } L \text { is a great fan of Person } \\
M \text {, who is a popular singer, actor } \\
\text { and talk-show celebrity. Person } M \\
\text { dies when a building collapses. }\end{array}$ & 0.4 & 0.7 & 1 & 1 & 1.2 \\
\hline & $\begin{array}{l}\text { Would person } L \text { have suicidal } \\
\text { thoughts? }\end{array}$ & & & & & \\
\hline & 1. No 2.Slight 3.Moderate 4.Strong & & & & & \\
\hline \multirow[t]{3}{*}{22} & $\begin{array}{l}\text { Person } \mathrm{L} \text { is a great fan of Person } \\
\mathrm{M} \text {, who is a popular singer, actor } \\
\text { and talk-show celebrity. Person } \mathrm{M} \\
\text { dies by jumping from a building. }\end{array}$ & 1 & 0.9 & 0.8 & 0.9 & 2.34 \\
\hline & $\begin{array}{l}\text { Would person } L \text { have suicidal } \\
\text { thoughts? }\end{array}$ & & & & & \\
\hline & 1. No 2.Slight 3.Moderate 4.Strong & & & & & \\
\hline \multirow[t]{3}{*}{23} & $\begin{array}{l}\text { Person N's parent has committed a } \\
\text { serious crime. Person } N \text { is aware of } \\
\text { the facts. Person } N \text { has been } \\
\text { subpoenaed to appear in court and } \\
\text { will be asked questions under oath, } \\
\text { which will probably lead to the } \\
\text { parent being convicted and } \\
\text { receiving a jail sentence. }\end{array}$ & 0.6 & 0.9 & 0.9 & 0.9 & 2.73 \\
\hline & $\begin{array}{l}\text { Would person } \mathrm{N} \text { have suicidal } \\
\text { thoughts? }\end{array}$ & & & & & \\
\hline & 1. No 2.Slight 3.Moderate 4.Strong & & & & & \\
\hline \multirow[t]{3}{*}{24} & $\begin{array}{l}\text { Person } O \text { is in love with Person } P \text {, } \\
\text { but person O's parents want Person } \\
O \text { to marry a third person, of their } \\
\text { choosing. }\end{array}$ & 1 & 0.9 & 0.9 & 0.9 & 2.8 \\
\hline & $\begin{array}{l}\text { Would person } \mathrm{O} \text { have suicidal } \\
\text { thoughts? }\end{array}$ & & & & & \\
\hline & 1. No 2.Slight 3.Moderate 4.Strong & & & & & \\
\hline
\end{tabular}




\begin{tabular}{|c|c|c|c|c|c|c|}
\hline No. & & CVR & $\begin{array}{l}\mathrm{CVI} \\
\text { (Relevance) }\end{array}$ & $\begin{array}{l}\text { CVI } \\
\text { (Simplicity) }\end{array}$ & $\begin{array}{l}\text { CVI } \\
\text { (Clarity) }\end{array}$ & $\begin{array}{l}\text { Impact } \\
\text { score }\end{array}$ \\
\hline \multirow[t]{3}{*}{25} & $\begin{array}{l}\text { Person Q has a serious gambling } \\
\text { problem, has lost the family's } \\
\text { savings and is in debt. Bills are } \\
\text { starting to arrive which cannot be } \\
\text { easily paid. }\end{array}$ & 1 & 1 & 1 & 1 & 3.44 \\
\hline & $\begin{array}{l}\text { Would person Q have suicidal } \\
\text { thoughts? }\end{array}$ & & & & & \\
\hline & 1. No 2.Slight 3.Moderate 4.Strong & & & & & \\
\hline \multirow[t]{3}{*}{26} & $\begin{array}{l}\text { Person Q has a serious gambling } \\
\text { problem, has lost the family's } \\
\text { savings and is deeply in debt. } \\
\text { Person Q's family is about to be } \\
\text { turned out onto the street by debt } \\
\text { collectors. }\end{array}$ & 1 & 0.9 & 1 & 1 & 3.44 \\
\hline & $\begin{array}{l}\text { Would person Q have suicidal } \\
\text { thoughts? }\end{array}$ & & & & & \\
\hline & 1. No 2.Slight 3.Moderate 4.Strong & & & & & \\
\hline \multirow[t]{3}{*}{27} & $\begin{array}{l}\text { Person } \mathrm{R} \text { cannot find work and is } \\
\text { having trouble paying the family } \\
\text { bills. }\end{array}$ & 1 & 1 & 1 & 1 & 2.8 \\
\hline & $\begin{array}{l}\text { Would person } \mathrm{R} \text { have suicidal } \\
\text { thoughts? }\end{array}$ & & & & & \\
\hline & 1. No 2.Slight 3.Moderate 4.Strong & & & & & \\
\hline \multirow[t]{3}{*}{28} & $\begin{array}{l}\text { Person R cannot find work and the } \\
\text { family is destitute. Person R's } \\
\text { family is about to be turned out } \\
\text { onto the street by debt collectors. }\end{array}$ & 1 & 1 & 1 & 1 & 3.78 \\
\hline & $\begin{array}{l}\text { Would person } \mathrm{R} \text { have suicidal } \\
\text { thoughts? }\end{array}$ & & & & & \\
\hline & 1. No 2.Slight 3.Moderate 4.Strong & & & & & \\
\hline \multirow[t]{3}{*}{29} & $\begin{array}{l}\text { Person S has a } 3 \text { year old child } \\
\text { with terminal (will be fatal) cancer. }\end{array}$ & 0.2 & 0.6 & 1 & 0.9 & 0.48 \\
\hline & $\begin{array}{l}\text { Would person } \mathrm{S} \text { have suicidal } \\
\text { thoughts? }\end{array}$ & & & & & \\
\hline & 1. No 2.Slight 3.Moderate 4.Strong & & & & & \\
\hline
\end{tabular}




\begin{tabular}{|c|c|c|c|c|c|c|}
\hline No. & & CVR & $\begin{array}{l}\mathrm{CVI} \\
\text { (Relevance) }\end{array}$ & $\begin{array}{l}\text { CVI } \\
\text { (Simplicity) }\end{array}$ & $\begin{array}{l}\text { CVI } \\
\text { (Clarity) }\end{array}$ & $\begin{array}{l}\text { Impact } \\
\text { score }\end{array}$ \\
\hline \multirow[t]{3}{*}{30} & $\begin{array}{l}\text { Person } U \text { is convicted of rape and } \\
\text { murder, and has been sentenced to } \\
\text { life in jail without parole. }\end{array}$ & 0.8 & 0.9 & 1 & 1 & 3.78 \\
\hline & $\begin{array}{l}\text { Would person U have suicidal } \\
\text { thoughts? }\end{array}$ & & & & & \\
\hline & 1. No 2.Slight 3.Moderate 4.Strong & & & & & \\
\hline \multirow[t]{3}{*}{31} & $\begin{array}{l}\text { Person } V \text { is the spouse of Person } U \\
\text { (the rapist-murderer in question } \\
\text { 33). }\end{array}$ & 0.6 & 0.9 & 0.9 & 0.9 & 2.1 \\
\hline & $\begin{array}{l}\text { Would person } V \text { have suicidal } \\
\text { thoughts? }\end{array}$ & & & & & \\
\hline & 1. No 2.Slight 3.Moderate 4.Strong & & & & & \\
\hline \multirow[t]{3}{*}{32} & $\begin{array}{l}\text { Person W had always been } \\
\text { popular. However, since winning a } \\
\text { prize, Person W has been subjected } \\
\text { to a sustained, malicious web } \\
\text { campaign, including accusations } \\
\text { of conceit, sexual deviance and } \\
\text { dishonest acts. }\end{array}$ & 0.6 & 0.5 & 0.9 & 0.8 & 1.65 \\
\hline & $\begin{array}{l}\text { Would person W have suicidal } \\
\text { thoughts? }\end{array}$ & & & & & \\
\hline & 1. No 2.Slight 3.Moderate 4.Strong & & & & & \\
\hline
\end{tabular}

\section{Reliability Results:}

In the reliability study, we examined the two domains of internal consistency and temporal stability. In the internal consistency, 151 respondents completed the questionnaire. Then we calculated Cronbach's alpha and McDonald's Omega coefficients as 0.94 and 0.943 , respectively, according to which the questionnaire has acceptable reliability in terms of internal consistency. In the temporal stability, we asked the 30 respondents to fill in the questionnaire two weeks later. The correlation was measured using the retest method, using intraclass correlation with a two-way mixed model and consistency type. The ICC was 0.998 (95\% Cl: 0.997-0.999). Furthermore, we conducted a paired T-test in which the test and retest scores means were $55.73(S D=21.36)$ and $56.53(S D=20.73)$, respectively ( $p$-value $=0.001)$. Therefore, it can be concluded that this questionnaire has acceptable reliability in terms of time stability.

\section{Construct Validity Results:}

We performed confirmatory factor analysis using $\mathrm{R}$ software version 4 that confirmed that the questionnaire has one dimension. The results of this study can be seen in Table 4. Only the SRMR index, 
which should be below 0.08 , has a slightly higher value, but the other recommended indices are within the significant range. Each question in the fitted model also had significant coefficients.

Table 4

Confirmatory factor analysis

\begin{tabular}{|lll|}
\hline Measure's name & Value & Cut-off for good fit \\
\hline$\chi^{2} / \mathrm{df}$ & 1.269 & $<3$ \\
\hline $\mathrm{CFI}$ & 0.978 & $\mathrm{CFI} \geq 0.90$ \\
\hline TLI & 0.986 & $\mathrm{TLI} \geq 0.95$ \\
\hline RMSEA & 0.042 & $\mathrm{RMSEA}<0.08$ \\
\hline SRMR & 0.092 & SRMR $<0.08$ \\
\hline
\end{tabular}

\section{Discussion:}

This study investigated the validity and reliability of the "Social Attitudes to Suicide dealing with Predicaments" questionnaire. We prepared the final Persian version of the questionnaire according to the obtained results, which has acceptable validity and reliability.

The Predicaments questionnaire was designed to assess the community's attitude toward suicide and the impact of specific circumstances on decision-making for suicide. Therefore, it can be used to compare social groups. In this questionnaire, the respondents confront imaginary characters facing a set of challenging situations [22]. We asked the Respondents to decide whether these characters experience suicidal thoughts and to what extent. Afterwards, we scored the answers, and each person's total score shows the suicidal attitude for each respondent.

Social attitudes link crises and life problems to suicide, which are severe and unfavourable conditions, such as severe mental disorders and terminal diseases. In such conditions, the person considers death the only solution [27]. At the same time, the level of adaptability of people in dealing with problems and crises is different. Consequently, the response of a person with a low level of adaptability to a simple problem can lead to suicide. The high scores of the Predicaments questionnaire in a group of individuals or a population indicate the extent to which that group or population will allow a person in crisis to have the right to consider suicide as the solution to that problem.

Moreover, it ultimately predicts a high suicide rate in that group. This questionnaire's scores show a direct relationship between suicide and suicide rate in a group or population. Hence, one may use this tool to prevent suicide worldwide [22].

Most questionnaires measuring attitudes toward suicide are designed for a specific social, age, or occupational group and, in general, for a particular target group. A major problem in measuring attitudes toward suicide is the mental nature of attitudes and their variability over time [20]. 
The attitude of society plays a vital role in one's beliefs in justifying oneself in ending their life [28]. An effective way to prevent suicide is to increase awareness of attitudes and taboos about suicide [29]. Various studies have identified deterrents to suicide, including religious belief and anxiety about death [30].

A critical feature of the Predicaments questionnaire that prioritises it over other similar ones is that it assesses the legitimacy of suicide by using empathy and judgmental emotions; the greater the complexity of the predicaments, the more people's attitudes soften toward suicide, and the more severe the predicament is, the more judgmental emotions are supposed to arise [22].

\section{Suggestions And Limitations:}

We suggest adding questions specific to the cultural context of Iranian society to this questionnaire in future studies. We recommend measuring individuals' attitudes toward suicide on a large scale in different parts of Iran and comparing the results between diverse populations with different suicide rates. Higher scores of this questionnaire are expected to be recorded in communities with higher suicide rates. Obviously, by conducting large-scale studies at the international level, one might obtain sufficient evidence to determine the underlying suicide risk factors.

One of the limitations of this study is the small sample size. Another limitation was that it had not measured the relationship between the questionnaire and other questionnaires measuring suicide.

\section{Conclusions:}

Overall, based on the results of this study, it is confirmed that the Persian version of the Predicaments questionnaire has acceptable content and face validity, temporal stability, and internal consistency. Therefore, this questionnaire can be used in future research on Iranian society.

Suicide is positively influenced by society's social and cultural attitudes that to what extent they assume it as an option in response to the hardships of life. This study proved that this questionnaire is a novel tool capable of measuring people's attitudes toward suicide, hence a proper measure to implement suicide prevention efforts.

\section{Declarations:}

\section{Ethics approval and consent to participate:}

We adhered to the Helsinki Statement and the Ethics Committee of the Iran University of Medical Sciences protocols in all stages of the research. All respondents' information was kept confidential by the researchers. This study was approved by the ethics committee of the Iran University of Medical Sciences with the ID code IR.IUMS.FMD.REC.1399.033. 


\section{Consent for publication:}

Not applicable

\section{Availability of data and materials:}

The datasets used and/or analysed during the current study are available from the corresponding author on reasonable request.

\section{Competing Interests:}

The authors declare no competing interest.

\section{Funding:}

The authors declare that they have not received any funding in any stages of this research.

\section{Authors' contributions:}

MS \& AR designed the study. AR, AK \& MT made substantial contribution in data gathering. AR, LJ, and YP conducted the analysis. AR drafted the manuscript. SKM, SAM, and AK reviewed the manuscript. AR and MS contributed to the finalizing of the manuscript. All authors read and approved the final manuscript

\section{Acknowledgements:}

The authors would like to thank the medical students of the Iran University of Medical Sciences for their cooperation. None of the experts examining face validity authored the manuscript.

\section{References:}

1. Sadock BJ, Sadock VA. Kaplan and Sadock's synopsis of psychiatry: Behavioral sciences/clinical psychiatry: Lippincott Williams \& Wilkins; 2011.

2. Organization WH. Preventing suicide: A global imperative. 2014.

3. Värnik P. Suicide in the world. International journal of environmental research and public health. 2012;9(3):760-71.

4. Ghoreyshi S, Mousavinasab S. Systematic review of researches on suicide and suicide attempt in Iran. 2008.

5. Mirhashemi S, Motamedi MHK, Mirhashemi AH, Taghipour H, Danial Z. Suicide in Iran. The Lancet. 2016;387(10013):29.

6. Fazel S, Runeson B. Suicide. N Engl J Med. 2020;382(3):266-74.

7. Bertolote JM, Fleischmann A. Suicide and psychiatric diagnosis: a worldwide perspective. World Psychiatry. 2002;1(3):181-5. 
8. Chang B, Gitlin D, Patel R. The depressed patient and suicidal patient in the emergency department: evidence-based management and treatment strategies. Emerg Med Pract. 2011;13(9):1-23; quiz -4.

9. Ajdacic-Gross V, Weiss MG, Ring M, Hepp U, Bopp M, Gutzwiller F, et al. Methods of suicide: international suicide patterns derived from the WHO mortality database. Bull World Health Organ. 2008;86(9):726-32.

10. Moradi SE. Evaluation of suicides resulting in death in Iran, comparing with the world rates. 2002.

11. Kiadaliri AA, Saadat S, Shahnavazi H, Haghparast-Bidgoli H. Overall, gender and social inequalities in suicide mortality in Iran, 2006-2010: a time trend province-level study. BMJ open. 2014;4(8).

12. Janghorbani M, Sharifirad GR. Completed and attempted suicide in Ilam, Iran (1995-2002): incidence and associated factors. 2005.

13. O'Neill S, O'Connor RC. Suicide in Northern Ireland: Epidemiology, risk factors, and prevention. The Lancet Psychiatry. 2020;7(6):538-46.

14. de la Garza ÁG, Blanco C, Olfson M, Wall MM. Identification of suicide attempt risk factors in a national US survey using machine learning. JAMA psychiatry. 2021;78(4):398-406.

15. Ji YD, Robertson FC, Patel NA, Peacock ZS, Resnick CM. Assessment of risk factors for suicide among US health care professionals. JAMA surgery. 2020;155(8):713-21.

16. Yip PS, Caine E, Yousuf S, Chang SS, Wu KC, Chen YY. Means restriction for suicide prevention. Lancet. 2012;379(9834):2393-9.

17. Ritzer G, Stepnisky J. The Wiley-Blackwell companion to major social theorists: John Wiley \& Sons; 2011.

18. Lester D. Suicide and islam. Arch Suicide Res. 2006;10(1):77-97.

19. Aggarwal N. Rethinking suicide bombing. Crisis. 2009;30(2):94-7.

20. Kodaka M, Poštuvan V, Inagaki M, Yamada M. A systematic review of scales that measure attitudes toward suicide. International journal of social psychiatry. 2011;57(4):338-61.

21. Strukcinskiene B, Strukcinskaite V, Rauckiene-Michaelsson A. The links between suicidal behaviour and endorsement of myths about suicide in young people. Social welfare: interdisciplinary approach. 2019;1(9):57-68.

22. Shahtahmasebi S, Varbanov S, Aleksandrov I, Pridmore S. Exploring social attitudes to suicide using a predicament questionnaire. Open journal of social sciences. 2016;4(12):58.

23. Brislin RW. Back-Translation for Cross-Cultural Research. Journal of Cross-Cultural Psychology. 1970;1(3):185-216.

24. Lawshe CH. A quantitative approach to content validity. Personnel psychology. 1975;28(4):563-75.

25. Waltz C, Bausell R. edition 1. Nursing research: Design, statistics and computer analysis Philadelphia FA Davis Co. 1983.

26. Yaghmaei F. Content validity and its estimation. 2003.

27. Shahtahmasebi S. De-politicizing youth suicide prevention. Frontiers in pediatrics. 2013;1:8. 
28. Diekstra RF, Kerkhof AJ. Attitudes toward suicide: development of a suicide attitude questionnaire (SUIATT). Current issues of suicidology: Springer; 1988. p. 462-76.

29. Osafo J, Knizek BL, Akotia CS, Hjelmeland H. Attitudes of psychologists and nurses toward suicide and suicide prevention in Ghana: A qualitative study. International Journal of Nursing Studies. 2012;49(6):691-700.

30. Minear JD, Brush LR. The correlations of attitudes toward suicide with death anxiety, religiosity, and personal closeness to suicide. OMEGA-Journal of Death and Dying. 1981;11(4):317-24. 